\title{
WEIGHTED SPACES OF HARMONIC AND HOLOMORPHIC FUNCTIONS: SEQUENCE SPACE REPRESENTATIONS AND PROJECTIVE DESCRIPTIONS
}

\author{
by PÄIVI MATTILA, EERO SAKSMAN and JARI TASKINEN \\ (Received 24th November 1994)
}

\begin{abstract}
We study the structure of inductive limits of weighted spaces of harmonic and holomorphic functions defined on the open unit disk of $\mathbb{C}$, and of the associated weighted locally convex spaces. Using a result of Lusky we prove, for certain radial weights on the open unit disk $D$ of $\mathbb{C}$, that the spaces of harmonic and holomorphic functions are isomorphic to complemented subspaces of the corresponding Köthe sequence spaces. We also study the spaces of harmonic functions for certain non-radial weights on $D$. We show, under a natural sufficient condition for the weights, that the spaces of harmonic functions on $D$ are isomorphic to corresponding spaces of continuous or bounded functions on $\partial D$.
\end{abstract}

AMS classification numbers: primary $46 \mathrm{E} 10$, secondary $46 \mathrm{~A} 13,46 \mathrm{E} 15$.

\section{Introduction}

We study the structure of inductive limits of weighted spaces of harmonic and holomorphic functions defined on the open unit disk of $\mathbb{C}$, and of the associated weighted locally convex spaces.

Weighted spaces and weighted inductive limits of spaces of holomorphic functions on open subsets of $\mathbb{C}^{N}(N \in \mathbb{N})$ arise in fields like linear partial differential operators, convolution equations, complex and Fourier Analysis and distribution theory. Weighted spaces of continuous functions have been studied in many works by $\mathrm{K}$. $\mathrm{D}$. Bierstedt, R. Meise, J. Bonet and others, see e.g. $[2,5-8,9-12,23]$, and as a consequence, this case is now quite well understood. In view of the recent counterexample of $\mathbf{J}$. Bonet and the third named author (see [13]), the corresponding spaces of holomorphic functions seem to be more complicated, and much less is known in that case; the same applies to the case of spaces of harmonic functions. It may be too hard to create a general theory of weighted inductive limits in the case of spaces of holomorphic or harmonic mappings which would be as satisfactory as the case of continuous mappings. So, it is natural to restrict the attention to some reasonable subclasses of weights.

In this paper we consider two cases. First, using the results of Lusky, [18], we study radial weights on the open unit disk $D$ of $\mathbb{C}$. We prove that, under certain conditions, the spaces of harmonic and holomorphic functions are isomorphic to complemented subspaces of the corresponding Köthe sequence spaces (Theorem 3.5). Second, we 
study the spaces of harmonic functions also for certain non-radial weights on $D$. Under a natural sufficient condition for the weights (Theorem 4.2) we show, using the Poisson kernel to solve the Dirichlet problem for Laplace operator, that the spaces of harmonic functions on $D$ are isomorphic to corresponding spaces of continuous or bounded functions on $\partial D$ (Corollaries 4.3 and 4.4, Theorem 4.7). The results imply immediate applications to the so called projective description and topological subspace problems.

\section{Preliminaries}

We denote by $\mathbb{R}^{+}$(resp. $\mathbb{R}_{0}^{+}$) the space of strictly positive reals (resp. $\mathbb{R}^{+} \cup\{0\}$ ). We denote $\mathbb{N}=\{1,2,3, \ldots\}$. $D$ denotes the open unit disk of $\mathbb{C}$. The vector spaces are over C. An isomorphism means an algebraic and topological isomorphism between locally convex spaces.

Let $V=\left(v_{k}\right)_{k=1}^{\infty}$ be a decreasing sequence of continuous strictly positive weight functions defined on $D$. We denote by $\mathcal{V} h(D)$ and $\mathcal{V} H(D)$ the inductive limits ind ${ }_{k} h v_{k}(D)$ and ind $H_{k} v_{k}(D)$, where $h v_{k}(D)$ (respectively, $H v_{k}(D)$ ) denotes the Banach space

$$
\begin{gathered}
\{f: D \rightarrow \mathbb{C} \text { harmonic (resp. holomorphic) } \mid \\
\left.p_{v_{k}}(f):=\sup _{z \in D} v_{k}(z)|f(z)|<\infty\right\} .
\end{gathered}
$$

In order to describe the topology of the weighted inductive limits Bierstedt, Meise and Summers [11] introduced the system of weights $\bar{V}$, associated with the sequence $V$,

$$
\bar{V}=\left\{\bar{v}: D \rightarrow \mathbb{R}^{+} \text {continuous } \mid \forall k \in \mathbb{N} \exists C_{k}>0 \text { such that } \bar{v} \leq C_{k} v_{k}\right\}
$$

The projective hull $h \bar{V}(D)$ (resp. $H \bar{V}(D)$ ) of $\mathcal{V} h(D)$ (resp. $\mathcal{V} H(D)$ ) is the locally convex space

$$
\begin{aligned}
&\{f: D \rightarrow \mathbb{C} \text { harmonic (resp. holomorphic) } \mid \\
&\left.p_{\bar{v}}(f):=\sup _{z \in D} \bar{v}(z)|f(z)|<\infty \text { for all } \bar{v} \in \bar{V}\right\}
\end{aligned}
$$

endowed with the locally convex topology defined by the seminorms $p_{\bar{v}}$ as $\bar{v}$ varies in $\bar{V}$. Clearly the inclusions $\mathcal{V} h(D) \hookrightarrow h \bar{V}(D)$ and $\mathcal{V} H(D) \hookrightarrow H \bar{V}(D)$ are continuous.

We also deal with spaces of harmonic and holomorphic functions vanishing on the boundary. The definitions are the same as above except that $h v_{k}$ is replaced by

$$
h\left(v_{k}\right)_{0}(D)=\left\{f: D \rightarrow \mathbb{C} \text { harmonic }\left|\lim _{r \rightarrow 1} \sup _{|z|=r} v_{k}(z)\right| f(z) \mid=0\right\}
$$

endowed with the norm $p_{v_{k}}$. The definition of $H\left(v_{k}\right)_{0}$ is analogous. The notation $\mathcal{V} h(D)$ (resp. $\mathcal{V} H(D), h \bar{V}(D), H \bar{V}(D))$ is replaced by $\mathcal{V}_{0} h(D)$ (resp. $\left.\mathcal{V}_{0} H(D), h \bar{V}_{0}(D), H \bar{V}_{0}(D)\right)$

We shall also need the corresponding space of continuous mappings. The definitions and notations are analogous, with $h$ replaced by $C$ and $D$, say, by a metric space $G$. 


\section{WEIGHTED SPACES OF HARMONIC AND HOLOMORPHIC FUNCTIONS}

For the basic properties of these spaces we refer to $[11]$ and $[9,10]$. In $[11]$ it was proved that $\mathcal{V} C(D)=C \bar{V}(D)$ and $\mathcal{V} H(D)=H \bar{V}(D)$ hold algebraically and that the two spaces in each equality have the same bounded sets. Moreover one of the main results in [11] shows that if $V$ satisfies condition $(S)$

(S) for all $k$ there is $l$ such that $\lim _{|z| \rightarrow 1} \frac{v_{1}(z)}{v_{k}(z)}=0$,

then $\mathcal{V} H(D)=H \bar{V}(D)$ holds topologically and $\mathcal{V} H(D)$ is a topological subspace of $\mathcal{V} C(D)$. In [10], [2] and [7] the topological identity $\mathcal{V} C(D)=C \bar{V}(D)$ was characterized in terms of a condition $(D)$ on the sequence $V$.

There is a special notation for the spaces of continuous mappings in the case $G=\mathbb{N}$ (discrete topology). In this case we denote (see [12]; [9, Section 4]) $\mathcal{V}_{0} C(G)$ (resp. $\left.\mathcal{V} C(G), C \bar{V}_{0}(G), C \bar{V}(G)\right)$ by $k_{0}=k_{0}(\mathbb{N}, V)$ (resp. $k_{\infty}=k_{\infty}(\mathbb{N}, V), K_{0}=K_{0}(\mathbb{N}, \bar{V})$, $\left.K_{\infty}=K_{\infty}(\mathbb{N}, \bar{V})\right)$. The linear topological properties of these spaces are particularly well understood, see [12], [5], [17] and [23].

We refer to [8] for a survey article on spaces of type $\mathcal{V} C(G)$.

\section{Radial weights on the unit disc}

In this section we study inductive limits of spaces $h v_{0}(D), H v_{0}(D), h v(D)$ and $H v(D)$, and the associated weighted spaces, in the case the weights $v$ are radial and satisfy certain technical conditions. Using the approach of Lusky, [18], we present these spaces as complemented subspaces of Köthe sequence spaces. Since the structure of Köthe spaces is nowadays well known, we can thus solve e.g. the projective description problem in some cases.

3.1. Notation. Unless otherwise stated, throughout this section we consider radial weights

$$
v: D \rightarrow] 0, \infty[
$$

which are continuous, non-increasing with respect to $|z|$, and satisfy $v(0)=1, v(r)>0$ for $0<r<1$ and $v\left(e^{i 0}\right):=\lim _{r \rightarrow 1} v\left(r e^{i 0}\right)=0$ for $0 \leq \theta \leq 2 \pi$.

If $f: D \rightarrow \mathbb{C}$, we denote

$$
p_{v}(f):=\sup _{z \in D} v(z)|f(z)|
$$

The following condition (*) for $v$ was introduced in [18], and will also play a role here:

$$
\inf _{n \in \mathbb{N}} \frac{v\left(1-2^{-n-1}\right)}{v\left(1-2^{-n}\right)}>0
$$


Let $f$ be a harmonic function $D \rightarrow \mathbb{C}$. Then by [16], I.A, $f$ has a representation which converges uniformly on compact subsets of $D$,

$$
f(z)=\sum_{k \geq 0}\left(\alpha_{k} z^{k}+\beta_{k} \bar{z}^{k}\right)
$$

where $z \in D$, and $\left(\alpha_{k}\right)_{k=1}^{\infty}$ and $\left(\beta_{k}\right)_{k=1}^{\infty}$ are sequences of complex numbers.

We denote for $n \in \mathbb{N}$ the Cesaro mean

$$
S_{n} f(z):=\sum_{k=0}^{n} \frac{n-k}{n}\left(\alpha_{k} z^{k}+\beta_{k} \bar{z}^{k}\right)
$$

We denote by $R_{n}$ the convolution with a de-la-Vallee-Poussin kernel: set $R_{0}:=0$ and, for $n \in \mathbb{N}$,

$$
R_{n} f(z):=\sum_{k=0}^{2^{n}}\left(\alpha_{k} z^{k}+\beta_{k} \bar{z}^{k}\right)+\sum_{k=2^{n}+1}^{2^{n+1}} \frac{2^{n+1}-k}{2^{n}}\left(\alpha_{k} z^{k}+\beta_{k} \bar{z}^{k}\right)
$$

Contrary to [18], we are also interested in the spaces without 0 -subindices. It will thus be necessary to present some preliminary results in a slightly more general form than in [18].

Lemma 3.2. There is a constant $c>0$ such that for every harmonic $f: D \rightarrow \mathbb{C}$ and $0<r<1$

$$
\begin{gathered}
\sup _{|z|=r}\left|R_{n} f(z)\right| \leq 3 \sup _{|z|=r}|f(z)| \text { for all } n \in \mathbb{N}, \\
\sup _{|z|=r}|f(z)| \leq \sup _{n \in \mathbb{N}} \sup _{|z|=r}\left|R_{n} f(z)\right|<\infty, \\
\sup _{|z|=r}\left|\left(R_{n}-R_{m}\right) f(z)\right| \leq c \sup _{|z|=r}\left|\left(R_{q}-R_{p}\right) f(z)\right|
\end{gathered}
$$

for $p \leq m \leq n \leq q$.

Proof. For $m$ large enough we have $R_{n} f_{m}=R_{n} f$, where $f_{m}(z):=\sum_{k=0}^{m}\left(\alpha_{k} z^{k}+\beta_{k} \bar{z}^{k}\right)$, see (3.3). Now (3.6) holds for $f_{m}$ in place of $f$, by [18, Section 3.1], hence, it holds also for $f$, since $f_{m} \rightarrow f$ uniformly on compact subsets on of $D$.

The first inequality of (3.7) follows approximately similarly the function $f$ by $f_{m}$ and using (3.6) to evaluate $R_{n}\left(f-f_{m}\right)$. The second inequality of (3.7) follows from (3.6).

The inequality (3.8) follows from [18, Section 3.1], since $R_{m} R_{n}=R_{\min (n, m)}$ for $m \neq n$.

The following lemma, in the case $f \in h v_{0}(D)$, is an important result by Lusky. We need to indicate here how to extend it to the more general case. 
Lemma 3.3. Assume that $v$ satisfies (*) and the condition

$$
\inf _{k \in \mathbb{N}} \limsup _{n \rightarrow \infty} \frac{v\left(1-2^{-n-k}\right)}{v\left(1-2^{-n}\right)}<1
$$

There exists $c>0$ such that for all harmonic $f: D \rightarrow \mathbb{C}$, for all $n$,

$$
p_{v}\left(\left(R_{n+1}-R_{n}\right) f\right) \leq c \sup _{|z|=1-2^{-n}}\left|\left(\left(R_{n+1}-R_{n}\right) f\right)(z)\right| v\left(1-2^{-n}\right)
$$

Moreover, if

$$
\sup _{n \in N} \sup _{|z|=1-2^{-n}}\left|\left(\left(R_{n+1}-R_{n}\right) f\right)(z)\right| v\left(1-2^{-n}\right)<\infty
$$

then $f \in h v(D)$ and

$$
p_{v}(f) \leq c \sup _{n \in \mathbb{N}} \sup _{|z|=1-2^{-n}}\left|\left(\left(R_{n+1}-R_{n}\right) f\right)(z)\right| v\left(1-2^{-n}\right)
$$

Proof. We have $R_{m} R_{n}=R_{\min (m, n)}$ for $m \neq n\left(\left[18\right.\right.$, Lemma 3.1]). Thus $\left(R_{n+1}-R_{n}\right) f=$ $\left(R_{n+1}-R_{n}\right) R_{n+2} f$ and hence (3.10) follows for $f$ since it holds for the trigonometric polynomial $R_{n+2} f$, by [18, formula (1) in Section 4].

Assume then that the harmonic $f$ satisfies (3.11). The inequality (3.7) implies, for $0<r<1$,

$$
\sup _{|z|=r}|f(z)| v(r) \leq \sup _{k \in \mathbb{N}} \sup _{|z|=r}\left|R_{k} f(z)\right| v(r) \leq \sup _{k \in \mathbb{N}} p_{v}\left(R_{k} f\right)
$$

Since every $R_{k} f$ is a trigonometric polynomial, [18, Theorem (ii)], yields for a constant $c>0$ and for every $k \in \mathbb{N}$

$$
p_{v}\left(R_{k} f\right) \leq c \sup _{n \in N} \sup _{|z|=1-2^{-n}}\left|\left(R_{n+1}-R_{n}\right)\left(R_{k} f\right)(z)\right| v\left(1-2^{-n}\right) .
$$

For all $k$

$$
\left(R_{n+1}-R_{n}\right) R_{k}= \begin{cases}R_{n+1}-R_{n}, & \text { if } n<k-1, \\ R_{n+1}\left(R_{n+1}-R_{n}\right), & \text { if } n=k-1, \\ R_{n}\left(R_{n+1}-R_{n}\right), & \text { if } n=k, \\ 0, & \text { if } n>k,\end{cases}
$$

This and (3.6) imply

$$
\sup _{|z|=1-2^{-n}}\left|\left(R_{n+1}-R_{n}\right)\left(R_{k} f\right)(z)\right| \leq 3 \sup _{|z|=1-2^{-n}}\left|\left(R_{n+1}-R_{n}\right) f(z)\right|
$$

for all $n, k$. The result now follows by combining (3.13), (3.14), (3.16) and (3.11). 
To be able to consider spaces of holomorphic mappings we recall that for a harmonic $f: D \rightarrow \mathbb{C}, f(z)=\sum_{k} \alpha_{k} z^{k}+\beta_{k} \bar{z}^{k}$, the Riesz projection $R$ is defined by

$$
R f(z)=\beta_{0}+\sum_{k} \alpha_{k} z^{k}
$$

if the sum on the right hand side converges uniformly on the compact sets of $D$.

Lemma 3.4. (i) $R R_{n}=R_{n} R$ for all $n \in \mathbb{N}$.

(ii) There exists a constant $c>0$ such that if $: D \rightarrow \mathbb{C}$ is harmonic, then

$$
\sup _{|z|=r}\left|R\left(R_{n+1}-R_{n}\right) f(z)\right| \leq c \sup _{|z|=r}|f(z)|
$$

for all $0<r<1$ and $n$.

Proof. (i) See [18, Lemma 3.1.(i)].

(ii) If $f$ and $n$ are given, choose $m>n+1$. Then $R_{m} f$ is a trigonometric polynomial, and hence [18, Lemma 3.1.(i) and (ii)], and our Lemma 3.2., (3.6), yield

$$
\begin{gathered}
\sup _{|z|=r}\left|R\left(R_{n+1}-R_{n}\right) f(z)\right|=\sup _{|z|=r}\left|R\left(R_{n+1}-R_{n}\right) R_{m} f(z)\right| \\
\leq c_{1} \sup _{|z|=r}\left|R_{m} f(z)\right| \leq 3 c_{1} \sup _{|z|=r}|f(z)| .
\end{gathered}
$$

We next state the main result of this section.

Theorem 3.5. Let $V=\left(v_{k}\right)_{k=1}^{\infty}$ be a decreasing sequence of weights on $D$ satisfying the conditions after (3.1). Assume moreover that for some $\varepsilon_{0}>0, n_{0} \in \mathbb{N}$, every $v_{k}$ satisfies the conditions

$$
\inf _{n \in \mathbb{N}} \frac{v_{k}\left(1-2^{-n-1}\right)}{v_{k}\left(1-2^{-n}\right)}>\varepsilon_{0}
$$

and

$$
\limsup _{n \rightarrow \infty} \frac{v_{k}\left(1-2^{-n-n_{0}}\right)}{v_{k}\left(1-2^{-n}\right)}<1-\varepsilon_{0}
$$

There is a sequence $\left(m_{n}\right)_{n=0}^{\infty}, m_{n} \in \mathbb{N}_{0}, m_{0}=0, m_{n}<m_{n+1}$ for all $n$, as follows. Denoting $U:=\left(u_{k}\right)_{k=1}^{\infty}, u_{k}: \mathbb{N} \rightarrow \mathbb{R}^{+}$,

$$
u_{k}(m):=v_{k}\left(1-2^{-n}\right) \quad m_{n-1}<m \leq m_{n},
$$

we have the following as complemented subspaces:

(i) $h \bar{V}_{0}(D) \subset K_{0}(\mathbb{N}, \bar{U})$

(ii) $h \bar{V}(D) \subset K_{\infty}(\mathbb{N}, \bar{U})$

(iii) $\mathcal{V}_{0} h(D) \subset k_{0}(\mathbb{N}, U)$ 
(iv) $\mathcal{V} h(D) \subset k_{\infty}(\mathbb{N}, U)$

(v) $H \bar{V}_{0}(D) \subset K_{0}(\mathbb{N}, \bar{U})$

(vi) $H \bar{V}(D) \subset K_{\infty}(\mathbb{N}, \bar{U})$

(vii) $\mathcal{V}_{0} H(D) \subset k_{0}(\mathbb{N}, U)$

(viii) $\mathcal{V} H(D) \subset k_{\infty}(\mathbb{N}, U)$.

Before proving this we give the following technical result.

Lemma 3.6. If $V$ is as in Theorem 3.5 and $\bar{w} \in \bar{V}$, then there exists a continuous, radial weight $\bar{v} \in \bar{V}$ on $D$ satisfying the following:

(i) $\bar{v} \geq c \bar{w}$ for a constant $c>0$,

(ii) the conditions after (3.1),

(iii) the condition (*), and

(iv) the condition (3.9).

Proof of Lemma 3.6. By (3.20), for every $k \in \mathbb{N}$ there exists an $n_{k}$ such that

$$
\frac{v_{k}\left(1-2^{-n-n_{0}}\right)}{v_{k}\left(1-2^{-n}\right)}<1-\varepsilon_{0} / 2
$$

for $n \geq n_{k}$; we may assume that $n_{k+1}>n_{k}$ for every $k \in \mathbb{N}$. Let the numbers $c_{k}>0$, $k \in \mathbb{N}$, be such that $\bar{w} \leq c_{k} v_{k}$. We choose the numbers $\alpha_{k}, k \in \mathbb{N}$,

$$
0<\alpha_{k}<2^{-k} c_{k}^{-1}
$$

as follows. Let $\alpha_{1}=1 /\left(4 c_{1}\right)$. If $\alpha_{k}$ is defined, we choose $\alpha_{k+1}$ so small that, in addition to $(3.23)$,

$$
\alpha_{k+1}\left(v_{k+1}(r)\right)^{-1} \leq \varepsilon_{0} 2^{-k-4} \min _{\substack{t \leq k \\ 0 \leq s \leq 1-2^{-n_{t+1}}}}\left\{\alpha_{t}\left(v_{t}(s)\right)^{-1}\right\}
$$

for all, $r, 0 \leq r \leq 1-2^{-n_{k+1}}$.

We define the radial, non-increasing weight function $\bar{v}$ on $D$ by

$$
\bar{v}^{\prime}(r):=\left(\sum_{k=1}^{\infty} \alpha_{k}\left(v_{k}(r)\right)^{-1}\right)^{-1}, \quad \bar{v}(r):=\bar{v}^{\prime}(r) / \bar{v}^{\prime}(0)
$$

for $0 \leq r<1$, and $\bar{v}(1)=0$. The weight $\bar{v}$ is continuous on $[0,1[$, since (3.24) implies that the series in (3.25) converges locally uniformly (set $t=0$ in (3.24)). Clearly, $\lim _{r \rightarrow 1} \bar{v}(r)=0$, hence $\bar{v}$ is continuous also on $[0,1]$. So, (ii) holds.

We have, for all $k \in \mathbb{N}$,

$$
\bar{v}^{\prime}(r) \leq\left(\alpha_{k}\left(v_{k}(r)\right)^{-1}\right)^{-1}=\alpha_{k}^{-1} v_{k}(r)
$$


and,

$$
\frac{1}{\bar{w}(r)} \geq \sup _{k} c_{k}^{-1}\left(v_{k}(r)\right)^{-1} \geq \sum_{k=1}^{\infty} 2^{-k} c_{k}^{-1}\left(v_{k}(r)\right)^{-1} \geq \sum_{k=1}^{\infty} \alpha_{k}\left(v_{k}(r)\right)^{-1},
$$

which implies

$$
\bar{w}(r) \leq\left(\sum_{k=1}^{\infty} \alpha_{k}\left(v_{k}(r)\right)^{-1}\right)^{-1}=\bar{v}^{\prime}(r) .
$$

So, (3.26) and (3.27) imply $\bar{v} \in \bar{V}$ and (i).

For every $n \in \mathbb{N}$, (3.19) yields

$$
\frac{\bar{v}\left(1-2^{-n-1}\right)}{\bar{v}\left(1-2^{-n}\right)}=\frac{\sum_{k=1}^{\infty} \alpha_{k}\left(v_{k}\left(1-2^{-n}\right)\right)^{-1}}{\sum_{k=1}^{\infty} \alpha_{k}\left(v_{k}\left(1-2^{-n-1}\right)\right)^{-1}} \geq \frac{\sum_{k=1}^{\infty} \varepsilon_{0} \alpha_{k}\left(v_{k}\left(1-2^{-n-1}\right)\right)^{-1}}{\sum_{k=1}^{\infty} \alpha_{k}\left(v_{k}\left(1-2^{-n-1}\right)\right)^{-1}}=\varepsilon_{0} .
$$

Hence, (*) holds for $\bar{v}$.

To prove (3.9) we assume that $K \in \mathbb{N}$ and that $n \in \mathbb{N}$ satisfies $n_{K} \leq n<n_{K+1}$. Using (3.24) we get

$$
\begin{aligned}
& \frac{\bar{v}\left(1-2^{-n-n_{0}}\right)}{\bar{v}\left(1-2^{-n}\right)}=\frac{\sum_{k=1}^{\infty} \alpha_{k}\left(v_{k}\left(1-2^{-n}\right)\right)^{-1}}{\sum_{k=1}^{\infty} \alpha_{k}\left(v_{k}\left(1-2^{-n-n_{0}}\right)\right)^{-1}} \\
& \quad \leq \frac{\sum_{k=1}^{K} \alpha_{k}\left(v_{k}\left(1-2^{-n}\right)\right)^{-1}+\sum_{k=K+1}^{\infty} \varepsilon_{0} 2^{-k-4} \alpha_{K}\left(v_{K}\left(1-2^{-n}\right)\right)^{-1}}{\sum_{k=1}^{K} \alpha_{k}\left(v_{k}\left(1-2^{-n-n_{0}}\right)\right)^{-1}} \\
& \quad \leq \frac{\left(1+\varepsilon_{0} / 8\right) \sum_{k=1}^{K} \alpha_{k}\left(v_{k}\left(1-2^{-n}\right)\right)^{-1}}{\sum_{k=1}^{K} \alpha_{k}\left(v_{k}\left(1-2^{-n-n_{0}}\right)\right)^{-1}}
\end{aligned}
$$

Since $n \geq n_{K}$, we can use (3.22) and (3.28) to estimate

$$
\frac{\bar{v}\left(1-2^{-n-n_{0}}\right)}{\bar{v}\left(1-2^{-n}\right)} \leq\left(1-\varepsilon_{0} / 2\right)\left(1+\varepsilon_{0} / 8\right)<1-\varepsilon_{0} / 4
$$

for $n_{K} \leq n<n_{K+1}$. Since $K$ is arbitrary, this implies (3.9)

Proof of Theorem 3.5. We first consider the case (i) (resp. (ii)). The following proof is analogous to the Banach space case considered in [18, proof of Lemma 3.8]. Because of the technical details it is necessary to repeat the whole proof.

Denote $r_{n}=1-2^{-n}$ and $\Gamma_{n}=\left\{z \in D|| z \mid=r_{n}\right\}$. Let $Y_{n}$ be the space of functions on $\Gamma_{n}$ which is the closure of trigonometric polynomials with respect to the sup-norm $\|f\|_{n}:=\sup _{|z|=r_{n}}|f(z)|$. Denoting by $h(D)$ the space of all harmonic functions $D \rightarrow \mathbb{C}$, a partition of unity argument yields a finite dimensional subspace $X_{n} \subset Y_{n}$ with

$$
\left.\left(R_{n+2}-R_{n-1}\right) h(D)\right|_{\Gamma_{n}} \subset X_{n}
$$


and

$$
\sup _{n} d\left(X_{n}, \ell_{\infty}^{\operatorname{dim}} X_{n}\right)<\infty
$$

where $d$ denotes the Banach-Mazur distance. We may assume that $X_{n}$ is spanned by trigonometric polynomials. We define the weight system $W=\left(w_{k}\right)_{k=1}^{\infty}$ on $\mathbb{N}$ by $w_{k}(n)=v_{k}\left(r_{n}\right)$, and

$$
\begin{gathered}
\Lambda_{0}=\left\{x:=\left(x_{n}\right)_{n=1}^{\infty} \mid x_{n} \in X_{n}, \quad q_{\bar{w}}(x):=\sup _{n \in \mathbb{N}} \bar{w}(n)\left\|x_{n}\right\|_{n}<\infty,\right. \\
\left.\lim _{n \rightarrow \infty} \bar{w}(n)\left\|x_{n}\right\|_{n}=0 \quad \text { for every } \bar{w} \in \bar{W}\right\} \\
\left(\text { resp. } \Lambda=\left\{x:=\left(x_{n}\right) \mid x_{n} \in X_{n}, \quad q_{\bar{w}}(x)<\infty \quad \text { for every } \bar{w} \in \bar{W}\right\}\right) .
\end{gathered}
$$

We show that $h \bar{V}_{0}(D)$ (resp. $h \bar{V}(D)$ ) is isomorphic to a complemented subspace of $\Lambda_{0}$ (resp. $\Lambda$ ). This is enough, since by $(3.30), \Lambda_{0}(\operatorname{resp} . \Lambda)$ is isomorphic to $K_{0}(\mathbb{N}, U)$ (resp. $K_{\infty}(\mathbb{N}, \bar{U})$ ), if we define in (3.21) (inductively)

$$
m_{1}:=\operatorname{dim} X_{1}, \quad m_{n+1}:=m_{n}+\operatorname{dim} X_{n+1}
$$

for $n \in \mathbb{N}$.

Define $T: h \bar{V}_{0}(D) \rightarrow \Lambda_{0}($ resp. $h \bar{V}(D) \rightarrow \Lambda$ ) by

$$
T f=\left(\left.\left(R_{n+2}-R_{n-1}\right) f\right|_{\Gamma_{n}}\right)_{n=1}^{\infty}
$$

where $R_{0}=0$. To show that $T$ is continuous, let $\bar{w} \in \bar{W}$ be arbitrary and let the numbers $c_{k}>0$ be such that $\bar{w} \leq c_{k} w_{k}$; since every $w_{k}$ is decreasing, we may also assume that $\bar{w}$ is decreasing. Define the radial, continuous weight function $\bar{v}^{\prime}$ on $D$ as follows. Define first $t:[0,1[\rightarrow[0,1[$ by

$$
t(r)=\left(r-r_{n}\right) /\left(r_{n+1}-r_{n}\right)
$$

if $r$ is such that $r_{n} \leq r<r_{n+1}$. Set then, for $n \in \mathbb{N}$, for $r_{n-1} \leq r<r_{n}$, where $r_{0}=0$,

$$
\bar{v}^{\prime}(r)=(1-t(r)) \bar{w}(n+1)+t(r) \bar{w}(n+2),
$$

and $\bar{v}^{\prime}(1)=0$, and extend $\bar{v}^{\prime}$ to $\bar{D}$ by radial symmetry. Since $\bar{w}$ is decreasing, it is easy to see that $\bar{v}^{\prime} \in \bar{V}$, and hence, we can choose the weight $\bar{v} \in \bar{V}, \bar{v} \geq c_{1} \bar{v}^{\prime}$, for a constant $c_{1}>0$, as in Lemma 3.6. Since $\bar{v}$ satisfies the condition $(*)$ and, by the definition of $\bar{v}^{\prime}$, the inequality $\bar{w}(n+1) \leq c_{1}^{-1} \bar{v}\left(r_{n}\right), n \in \mathbb{N} \cup\{0\}$, we find a $c>0$ such that $\bar{w}(n) \leq c \bar{v}\left(r_{n}\right)$ for all $n \in \mathbb{N}$. This and (3.6) imply

$$
\begin{aligned}
& q_{\bar{w}}(T f)=\sup _{n \in \mathbb{N}} \sup _{|z|=r_{n}}\left|\left(R_{n+2}-R_{n-1}\right) f(z)\right| \bar{w}(n) \\
& \leq 6 c \sup _{n \in \mathbb{N}} \sup _{|z|=r_{n}}|f(z)| \bar{v}\left(r_{n}\right) \leq 6 c \sup _{z \in D}|f(z)| \bar{v}(z)
\end{aligned}
$$


and so $T$ is continuous; a similar estimate also shows that, for $f \in h \bar{V}_{0}(D)$,

$$
\sup _{|z|=r_{n}}\left|\left(R_{n+2}-R_{n-1}\right) f(z)\right| \bar{w}(n) \rightarrow 0 \quad \text { as } n \rightarrow \infty,
$$

hence, $T\left(h \bar{V}_{0}(D)\right) \subset \Lambda_{0}$ in case (i).

As in [18, (1) in the proof of Lemma 3.8], a left inverse of $T$ can be defined by

$$
S:\left(x_{n}\right)_{n=1}^{\infty} \mapsto R_{1} \hat{x}_{1}+\sum_{n=1}^{\infty}\left(R_{n+1}-R_{n}\right) \hat{x}_{n},
$$

where $x_{n} \in X_{n}$ and $\hat{x}_{n}$ is the canonical extension of $x_{n}$ to a trigonometric polynomial on $D$.

We must first show that the right hand side of (3.36) converges uniformly on compact subsets of $D$. This can be done, using [18], as follows. It is possible to choose a radial, continuous, non-increasing weight $v$, satisfying the conditions (*) and (3.9), such that $h \bar{V}(D) \subset h v_{0}(D)$ algebraically. (For example, fix first $\bar{v} \in \bar{V}$ satisfying (*) etc., and define then $v(r):=(1-r) \bar{v}(r)$.) Define for all $n \in \mathbb{N}$

$$
\omega(n)=v\left(r_{n}\right)
$$

and denote by $\Lambda_{\omega}$ the space

$$
\Lambda_{\omega}=\left\{x:=\left(x_{n}\right) \mid x_{n} \in X_{n}, \lim _{n \rightarrow \infty} \omega(n)\left\|x_{n}\right\|_{n}=0\right\}
$$

We then also have $\Lambda \subset \Lambda_{\omega}$. If $\Lambda_{\omega}$ is endowed with the norm $\sup _{n} \omega(n)\left\|x_{n}\right\|_{n}$, then by [18, proof of Lemma 3.8], $S: \Lambda_{\omega} \rightarrow h \omega_{0}(D)$ is a well-defined, continuous operator. Hence, $S x$ is a harmonic function on $D$ for all $x \in \Lambda \subset \Lambda_{\omega}$, and the series in (3.36) converges uniformly on compact subsets of $D$.

It remains to prove that $S\left(\Lambda_{0}\right) \subset h \bar{V}_{0}(D)$ (resp. $S(\Lambda) \subset h \bar{V}(D)$ ) and that $S$ is continuous. Let $\bar{v}^{\prime} \in \bar{V}$ and choose $\bar{v} \in \bar{V}, \bar{v} \geq c \bar{v}^{\prime}$ as in Lemma 3.6. Define $\bar{w}(n)=\bar{v}\left(r_{n}\right)$ for $n \in \mathbb{N}$; clearly, $\bar{w} \in \bar{W}$. We want to use Lemma 3.4 , and we thus need to estimate the expression

$$
\begin{aligned}
& \sup _{n \in \mathbb{N}} \sup _{|z|=r_{n}}\left|\left(R_{n+1}-R_{n}\right) S x(z)\right| \bar{v}\left(r_{n}\right) \leq \sup _{n \in \mathbb{N}} \sup _{|z|=r_{n}}\left|\left(R_{n+1}-R_{n}\right) R_{1} \hat{x}_{1}(z)\right| \bar{v}\left(r_{n}\right) \\
& \quad+\sup _{n \in \mathbb{N}} \sup _{|z|=r_{n}}\left|\sum_{t=1}^{\infty}\left(R_{n+1}-R_{n}\right)\left(R_{t+1}-R_{t}\right) \hat{x}_{t}(z)\right| \bar{v}\left(r_{n}\right),
\end{aligned}
$$

where $x=\left(x_{n}\right)_{n=1}^{\infty} \in \Lambda$.

Since $\hat{x}_{1}$ is a trigonometric polynomial, we have by [18, Lemma 3.1$]$

$$
\begin{aligned}
& \sup _{n \in N} \sup _{|z|=r_{n}}\left|\left(R_{n+1}-R_{n}\right) R_{1} \hat{x}_{1}(z)\right| \bar{v}\left(r_{n}\right)=\sup _{|z|=r_{1}}\left|\left(R_{2}-R_{1}\right) R_{1} \hat{x}_{1}(z)\right| \bar{v}\left(r_{1}\right) \\
& \quad \leq c \sup _{|z|=r_{1}}\left|x_{1}(z)\right| \bar{v}\left(r_{1}\right) \leq c q \bar{w}(x)<\infty
\end{aligned}
$$


for some constants $c>0$.

We also have

$$
\left(R_{n+1}-R_{n}\right)\left(R_{t+1}-R_{t}\right)= \begin{cases}\left(R_{n}-R_{n-1}\right)\left(R_{n+1}-R_{n}\right), & \text { if } t=n-1 \\ \left(R_{n+1}-R_{n}\right)^{2}, & \text { if } t=n, \\ \left(R_{n+2}-R_{n+1}\right) R_{n+1}, & \text { if } t=n+1 \\ 0, & \text { for other } t\end{cases}
$$

since $R_{t} R_{n}=R_{\min (t, n)}$ for $t \neq n$. Hence, the second term on the right hand side of (3.38) can be estimated by $(\hat{x}:=0)$

$$
\sup _{n \in \mathbb{N}} p_{\bar{v}}\left(\left(R_{n}-R_{n-1}\right)\left(R_{n+1}-R_{n}\right) \hat{x}_{n-1}\right)+\sup _{n \in \mathbb{N}} p_{\bar{v}}\left(\left(R_{n+1}-R_{n}\right)^{2} \hat{x}_{n}\right)+\sup _{n \in \mathbb{N}} p_{\bar{v}}\left(\left(R_{n+2}-R_{n+1}\right) R_{n+1} \hat{x}_{n+1}\right) .
$$

We use Lemma 3.3 and then Lemma 3.2 to estimate (3.41) by

$$
\begin{aligned}
& c \sup _{n \in \mathbb{N}} \sup _{|z|=r_{n-1}}\left|\left(R_{n}-R_{n-1}\right)\left(R_{n+1}-R_{n}\right) \hat{x}_{n-1}(z)\right| \bar{v}\left(r_{n-1}\right) \\
& \quad+c \sup _{n \in \mathbb{N}} \sup _{|z|=r_{n}}\left|\left(R_{n+1}-R_{n}\right)\left(R_{n+1}-R_{n}\right) \hat{x}_{n}(z)\right| \bar{v}\left(r_{n}\right) \\
& \quad+c \sup _{n \in \mathbb{N}} \sup _{|z|=r_{n+1}}\left|\left(R_{n+2}-R_{n+1}\right) R_{n+1} \hat{x}_{n+1}(z)\right| \bar{v}\left(r_{n+1}\right) \\
& \leq c \sup _{n \in \mathbb{N}} \sup _{|z|=r_{n-1}}\left|\hat{x}_{n-1}(z)\right| \bar{v}\left(r_{n-1}\right)+c_{1} \sup _{n \in \mathbb{N}} \sup _{|z|=r_{n}}\left|\hat{x}_{n}(z)\right| \bar{v}\left(r_{n}\right) \\
& \quad+c_{1} \sup _{n \in \mathbb{N}} \sup _{|z|=r_{n+1}}\left|\hat{x}_{n+1}(z)\right| \bar{v}\left(r_{n+1}\right) \leq c_{2} q_{\bar{w}}(x)<\infty .
\end{aligned}
$$

Hence, Lemma 3.3 and (3.38)-(3.42) imply $S(\Lambda) \subset h \bar{V}(D)$ in the case (ii) and the continuity of $S$ :

$$
p_{\bar{\nu}}(S x) \leq c \sup _{n \in \mathbb{N}} \sup _{|z|=r_{n}}\left|\left(R_{n+1}-R_{n}\right) S x(z)\right| \bar{v}\left(r_{n}\right) \leq c q_{\bar{w}}(x)
$$

If $x \in \Lambda_{0}$, then $S x \in h \bar{V}(D)$ can be approximated as well as we wish by elements of the form $S x^{(m)}$, where $x_{n}^{(m)}=x_{n}$ for $n \leq m$ and $x_{n}^{(m)}=0$ for $n>m$. But $S x^{(m)}$ is a trigonometric polynomial; hence $S \Lambda_{0}$ is contained in the closure of the subspace of trigonometric polynomials in $h \bar{V}(D)$. This implies $S\left(\Lambda_{0}\right) \subset h \bar{V}_{0}(D)$, see [21].

We have $\left(R_{n+1}-R_{n}\right)\left(R_{n+2}-R_{n-1}\right)=R_{n+1}-R_{n}$, see [18, Lemma 3.1], and on the other hand, $R_{1} f+\sum_{n=1}^{\infty}\left(R_{n+1}-R_{n}\right) f=f$ for $f \in h \bar{V}(D)$. Hence, $S T$ is the identity operator on $h \bar{V}_{0}(D)$ (resp. $h \bar{V}(D)$ ), and $T S$ is a continuous projection from $\Lambda_{0}$ onto a subspace isomorphic to $h \bar{V}_{0}(D)$ (resp. $\Lambda, h \bar{V}(D)$ ).

The cases (v) and (vi). Let $\bar{v}^{\prime} \in \bar{V}$ and let $\bar{v}, \bar{w}$ be as just above (3.38). The operator $S: \Lambda \rightarrow h \bar{V}(D)$ is a surjection, since $S T$ is the identity operator on $h \bar{V}(D)$. This and (3.43) imply

$$
p_{\bar{v}}(f) \leq \varrho_{\bar{v}}(f):=c \sup _{n \in \mathbb{N}} \sup _{|z|=r_{n}}\left|\left(R_{n+1}-R_{n}\right) f(z)\right| \bar{v}\left(r_{n}\right)<\infty
$$


for all $f \in h \bar{V}(D)$. On the other hand (3.43) also shows that $\varrho_{\bar{v}}(f) \leq c q_{\bar{w}}(T f)$, and since $T$ is contnuous, there exists a $\bar{v}^{\prime \prime} \in \bar{V}$ such that $q_{\bar{w}}(T f) \leq C p_{\bar{v}^{\prime \prime}}(f)$. We have thus shown that the system $\left(\varrho_{\bar{v}}\right)_{\bar{v} \in \bar{V}}$ defines the topology of the spaces $h \bar{V}_{0}(D)$ and $h \bar{V}(D)$. Using the representation (3.44) and Lemma 3.4 it is easy to see that the Riesz projection is continuous on $h \bar{V}_{0}(D)$ and $h \bar{V}(D)$. Hence, $H \bar{V}_{0}(D) \subset h \bar{V}_{0}(D)$ and $H \bar{V}(D) \subset h \bar{V}(D)$ as complemented subspaces.

The cases (iii) and (iv). Let us fix $k \in \mathbb{N}$. The proof of the case (i) shows that $h\left(v_{k}\right)_{0}$ is isomorphic to a complemented subspace of (cf. (3.31))

$$
\begin{gathered}
c_{0}\left(v_{k}\right):=\left\{x:=\left(x_{n}\right) \mid x_{n} \in X_{n}, \quad q_{v_{k}}(x):=c \sup _{n \in \mathbb{N}} w_{k}(n)\left\|x_{n}\right\|_{n}<\infty,\right. \\
\lim _{n \rightarrow \infty} w_{k}(n)\left\|x_{n}\right\|_{n}=0,
\end{gathered}
$$

where $w_{k}(n):=v_{k}\left(r_{n}\right)$. (One can verify this directly or define a new system of weights on $D, V^{\prime}:=\left(v_{t}^{\prime}\right)_{t=1}^{\infty}, v_{t}^{\prime}:=v_{k}$ for all $t$, and use $V^{\prime}$ instead of $V$ in the proof of (i).) What is important here is that this isomorphism is determined by the mappings $S$ and $T$ which do not depend on the weight $v_{k}$, see (3.34) and (3.36). So, since $S$ and $T$ are continuous mappings between the corresponding step spaces of $\mathcal{V}_{0} h(D)$ and ind ${ }_{k} c_{0}\left(v_{k}\right)$, they are also continuous between the inductive limits. Hence,

$$
\mathcal{V}_{0} h(D) \subset \operatorname{ind}_{k} c_{0}\left(v_{k}\right)
$$

as a complemented subspace, and we are done, since $\operatorname{ind}_{k} c_{0}\left(v_{k}\right)$ is isomorphic to $k_{0}(\mathbb{N}, U)$, see the remark connected with (3.33).

The case (iv) can be obtained in the same way using (ii).

Remark. The case (iii) follows also directly from [18, Theorem (ii),c].

(vii) and (viii). Analogously to (v) and (vi) we can see that the Riesz projection is continuous on the step spaces of $\mathcal{V}_{0} h(D)$ and $\mathcal{V} h(D)$, which implies the result. Again, (vii) could be obtained directly from the results of [18].

As we mentioned in Section 2, the structure of the Köthe spaces is well-known, and, together with our Theorem 3.5, can be used to solve the projective description problem and the topological subspace problem.

Theorem 3.7. Let $V$ and $U$ be as in Theorem 3.5, and assume that $U$ satisfies the condition $(D)$ (see [10, Section 2]). Then

(i) the spaces $h \bar{V}(D)$ and $H \bar{V}(D)$ are bornological, and $h \bar{V}(D) \cong \mathcal{V} h(D)$ and $H \bar{V}(D) \cong \mathcal{V} H(D)$ as algebraic and topological isomorphisms,

(ii) $\mathcal{V} H(D) \subset \mathcal{V} h(D) \subset \mathcal{V} C(D)$ as topological subspaces.

Proof. Since the condition $(D)$ holds for $U$, the space $K_{\infty}(\mathbb{N}, \bar{U})$ is bornological, [10, Theorem 2.3]. Since a complemented subspace of a bornological space is borno- 
logical, Theorem 3.5 implies the first part of (i). The second part follows from this.

It is well-known that (i) now implies (ii), see e.g. [13].

3.8. Examples. The systems $V=\left(v_{k}\right)_{k=1}^{\infty}$, where $v_{k}(1-r)=r^{x k /(k+1)}$ or $v_{k}(1-r)=$ $r^{2}(-\log (r / e))^{-k /(k+1)}$ and $\alpha \in \mathbb{R}^{+}$, satisfy the conditions of Theorem 3.5. Both of these examples are Schwartz spaces, as can be seen from the definition of the weight system $U$.

We also refer to [18, Examples after Theorem in Section 2].

One can construct more complicated examples using the following result. The idea of the example is to show that a space $h \bar{V}(D)$ can contain quite general Köthe sequence spaces as complemented subspaces, even if the weights are very nice.

Proposition 3.9. Let $W^{\prime}=\left(w_{k}^{\prime}\right)_{k=1}^{\infty}, w_{k}^{\prime}: \mathbb{N} \rightarrow \mathbb{R}^{+}$, be such that, for some $\varepsilon_{0}>0$ and $n_{0} \in \mathbb{N}$, every $w_{k}^{\prime}$ satisfies

(i) $w^{\prime}(1)=1, w_{k}^{\prime}(n+1) \leq w_{k}^{\prime}(n)$ for all $n$,

(ii)

(iii)

$$
\inf _{n \in \mathbb{N}} w_{k}^{\prime}(n+1) / w_{k}^{\prime}(n)>\varepsilon_{0},
$$

$$
\limsup _{n \rightarrow \infty} w_{k}^{\prime}\left(n+n_{1}\right) / w_{k}^{\prime}(n)<1-\varepsilon_{0}
$$

Then $K_{\infty}\left(\mathbb{N}, \bar{W}^{\prime}\right)$ is isomorphic to a complemented subspace of a space $h \bar{V}(D)$, where $V$ satisfies the conditions of Theorem 3.5.

Sketch of the proof. We first define the weight system $W=\left(w_{k}\right)_{k=1}^{\infty}, w_{k}: \mathbb{N} \rightarrow \mathbb{R}^{+}$, by

$$
w_{k}(5 n-t):=w_{k}^{\prime}(n) \text { for all } n \in \mathbb{N}, \quad t=0,1,2,3,4 .
$$

Using (i)-(iii) it is easy to see that for some $n_{1} \geq 5$ every $w_{k}$ satisfies

$1^{\circ} w_{k}(n+1) \leq w_{k}(n)$ for all $n$,

$2^{\circ} \inf _{n \in \mathrm{N}} w_{k}(n+1) / w_{k}(n)>\varepsilon_{0}$,

$3^{\circ} \lim \sup _{n \rightarrow \infty} w_{k}\left(n+n_{1}\right) / w_{k}(n)<1-\varepsilon_{0}$.

We choose the spaces $X_{n}$ as in the proof of Theorem 3.5 - they do not depend on the weights. We define the numbers $m_{n}, n \in \mathbb{N}_{0}$, as in (3.33), and then the space

$$
\Lambda=\left\{x:=\left(x_{n}\right) \mid x_{n} \in X_{n}, \quad q_{\bar{w}}(x):=\sup _{n \in \mathbb{N}} \bar{w}(n)\left\|x_{n}\right\|_{n}<\infty, \quad \text { for every } \bar{w} \in \bar{W}\right\}
$$

We define the weights $v_{k}$ by $v_{k}(0)=1, v_{k}\left(r_{n}\right)=w_{k}(n)$ for $n \in \mathbb{N}\left(r_{n}=1-2^{-n}\right)$, extend $v_{k}$ affinely to $\left[0,1\left[\right.\right.$ and then by radial symmetry to $D$. That $V=\left(v_{k}\right)$ satisfies the assumptions of Theorem 3.5 follows from $1^{\circ}-3^{\circ}$. Because of the similarity of the definitions (3.32) and (3.48), the proof of Theorem 3.5.(ii), shows that $T$ (defined in (3.34)) is an isomorphic embedding of $h \bar{V}(D)$ into $\Lambda$.

Denote now by $P_{n}$ the canonical projection from $\Lambda$ onto the $n$th coordinate space $X_{n}$. If we can find for each $n \in \mathbb{N}$ a non-zero vector

$$
e_{n} \in \bigoplus_{t=5 n-4}^{5 n} P_{t}(\Lambda)
$$


such that $e_{n} \in T(h \bar{V}(D))$, we are done: the subspace $E:=\overline{\mathrm{sp}_{n \in \mathrm{N}} e_{n}}$ is clearly complemented in $\Lambda$ and on the other hand isomorphic to $K_{\infty}\left(\mathbb{N}, \bar{W}^{\prime}\right)$. (See the relation between the weights $w_{k}^{\prime}$ and $w_{k}$.)

We define

$$
e_{n}:=T z^{2^{5 n-1}} .
$$

Then (3.49) holds, by the definition of $T$, since $R_{m} z^{2^{5 n-1}}=0$ for $m \leq 5 n-3$ and $R_{m} z^{2^{5 n-1}}=z^{2^{5 n-1}}$ for $m \geq 5 n$.

We leave it to the reader to consider Proposition 3.9 for the other spaces occurring in (i)-(viii) of Theorem 3.5.

\section{The case of non-radial weights and harmonic functions}

The idea of this section is to study whether a given weighted space of harmonic functions on $D$ is isomorphic to some standard weighted space of bounded or continuous functions on $\partial D$.

We start by some notation and definitions. Throughout this section we consider (not necessarily radial) weight functions $v: D \rightarrow] 0, \infty[$ which have continuous extensions $v: \bar{D} \rightarrow[0, \infty]$, where the half line is endowed with the usual compact topology. We write $v^{*}:=\left.v\right|_{\partial D}$, and we always assume that the sets

$$
N_{v}:=\left\{z \in \partial D \mid v^{*}(z)=0\right\}, \quad S_{v}:=\left\{z \in \partial D \mid v^{*}(z)=\infty\right\}
$$

are of measure zero.

For functions $f: D \rightarrow \mathbb{C}$ we denote $f_{r}\left(e^{i 0}\right)=f\left(r e^{i 0}\right)$. If $f$ has radial limits a.e. in $\partial D$, we define $f^{*}\left(e^{i 0}\right)=\lim _{r \rightarrow 1} f\left(r e^{i 0}\right)$ for $e^{i 0} \in \partial D$.

Assume that $f: \partial D \rightarrow \mathbb{C}$ is integrable and $z \in D$. We denote by $P(f, z)$ the value at $z$ of the usual (harmonic) Poisson extension of $f$, see [20, Section 11.7].

We still introduce some function spaces. We denote by $h v_{c}(D)$ the closed subspace of $h v(D)$ spanned by functions that admit continuous extensions to $\partial D$. If $V:=\left(v_{k}\right)_{k=1}^{\infty}$ is a decreasing sequence of weights as above, we denote $\mathcal{V}_{c} h(D):=\operatorname{ind}_{k} h\left(v_{k}\right)_{c}(D)$. The associated weighted space $h \bar{V}_{c}(D)$ is defined as the closed subspace of $h \bar{V}(D)$ spanned by elements which have a continuous extension to $\partial D$. In the following we study the relation of $\mathcal{V}_{c} h(D)$ and $h \bar{V}_{c}(D)$ only indirectly, by giving a sufficient condition for them to be isomorphic.

If $\Omega=D$ or $\partial D$ and $w$ is a weight on $\Omega$, we set

$$
L^{\infty} w(\Omega)=\left\{f: \partial D \rightarrow \mathbb{C} \text { measurable, } \mid \text { ess. } \sup _{z \in \Omega} w(z)|f(z)|<\infty\right\}
$$

with the usual identification of those functions that differ only on sets of measure zero and with the evident choice of norm; here "ess" refers to the two (resp. one) dimensional Lebesgue measure, if $\Omega=D$ (resp. $\partial D$ ). If $V=\left(v_{k}\right)_{k=1}^{\infty}$ is as above, we denote $V^{*}:=\left(v_{k}^{*}\right)_{k=1}^{\infty}, \mathcal{V}^{*} L^{\infty}(\partial D):=\operatorname{ind}_{k} L^{\infty} v_{k}^{*}(\partial D)$, and 


\section{WEIGHTED SPACES OF HARMONIC AND HOLOMORPHIC FUNCTIONS 55}

$$
L^{\infty} \overline{V^{*}}(\partial D):=\left\{f: \partial D \rightarrow \mathbb{C} \text { measurable } \mid \text { ess. } \sup _{z \in \partial D} \overline{v^{*}}(z)|f(z)|<\infty \text { for all } \overline{v^{*}} \in \overline{V^{*}}\right\}
$$

The Banach space $C^{*} v(\partial D)$ (in general not equal e.g. to $C v^{*}\left(\partial D \backslash N_{v}\right)$ ) is defined as the closure of $C(\partial D) \cap L^{\infty} v^{*}(\partial D)$ in $L^{\infty} v^{*}(\partial D)$.

Lemma 4.1. (i) Let $X$ denote the closed subspace of $L^{\infty} v(D)$ spanned by continuous functions belonging to $L^{\infty} v(D)$ and admitting a continuous extension to $\bar{D}$. Then $C^{*} v(\partial D)=\left\{g^{*} \mid g \in X\right\}$.

(ii) Let $f: \partial D \rightarrow \mathbb{C}$. Then $f \in C^{*} v(\partial D)$ if and only if (modulo sets of measure zero; recall that $f$ is in an $L^{\infty}$-type space) $\left.f v^{*}\right|_{\partial D \backslash\left(S_{0} \cup N_{v}\right)}$ is continuous and $\lim _{z \rightarrow z_{0}} f v^{*}(z)=0$ for all $z_{0} \in N_{v}$.

(iii) If $S_{v}=\emptyset$, then $C^{*} v(\partial D)$ is canonically isometric to $C\left(v^{*}\right)_{0}\left(\partial D \backslash N_{v}\right)$ (see Section 2).

(iv) If $N_{v}=\emptyset$, then $C^{*} v(\partial D)$ is canonically isometric to $\mathbb{C}_{v^{*}}\left(\partial D \backslash S_{v}\right)$.

Proof. (i) It is evident that each $g \in X$ has radial boundary values a.e. and that $g^{*} \in C^{*} v(\partial D)$. Conversely, assume that $f \in C^{*} v(\partial D)$. For $h \in C(\partial D) \cap L^{\infty} v^{*}(\partial D)$ define $\phi(h): \bar{D} \rightarrow \mathbb{C}$ by setting

$$
\phi(h)\left(r e^{i 0}\right)=\frac{h\left(e^{i 0}\right)}{\left|h\left(e^{i 0}\right)\right|} \min \left\{r\left|h\left(e^{i 0}\right)\right|, \frac{\|h\|_{L^{\infty} v^{*}(\partial D)}}{v\left(r e^{i 0}\right)}\right\}
$$

where we agree that $h\left(e^{i 0}\right) /\left|h\left(e^{i 0}\right)\right|=0$ if $h\left(e^{i \theta}\right)=0$. It is easily verified that $\phi(h) \in C(\bar{D})$

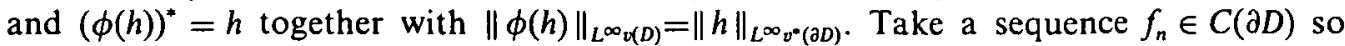
that $\left\|f-f_{n}\right\|_{L^{\infty} \nu^{*}(\partial D)} \leq 2^{-n}$ for $n \in \mathbb{N}$ and set $g=\phi\left(f_{1}\right)+\sum_{n=1}^{\infty} \phi\left(f_{n+1}-f_{n}\right)$. It follows that $g \in X$ and $g^{*}=f$.

(ii) "iff": Let $f \in C^{*} v(\partial D)$. Choose $f_{n} \in C(\partial D)$ so that $f_{n} \rightarrow f$ in the norm of $C^{*} v(\partial D)$. Now $f_{n} v^{*}$ is continuous on $\partial D \backslash S_{v}$ and $\left.f_{n} v^{*}\right|_{N_{v}}=0$ for each $n$. The claimed properties of $f$ follow at once as we note that $f_{n} v^{*}$ converges uniformly to $f v^{*}$ (with respect to the usual sup-norm) on $\partial D \backslash S_{v}$.

"if": Assume that $f$ satisfies the stated conditions. For $\varepsilon>0$ write $\left(N_{v}\right)_{\varepsilon}=$

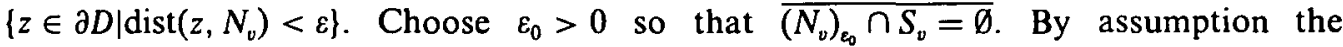
function $g:=\left.f v^{*}\right|_{\partial D \backslash\left(S_{v} \cup N_{v}\right)}$ has a continuous extension $\tilde{g}$ to $\partial D \backslash S_{v}$ so that $\left.\tilde{g}\right|_{N_{0}}=0$. Hence, by continuity, we may choose for each $n \in \mathbb{N}$ a number $\varepsilon_{n} \in\left[0, \varepsilon_{0}\right]$ with the property $|\tilde{g}(z)| \leq 1 / n$ for $z \in\left(N_{v}\right)_{\varepsilon_{n}}$. Set $f_{n}(z)=f(z) \min \left\{1,2 \varepsilon_{n}^{-1} \operatorname{dist}\left(z,\left(N_{v}\right)_{\varepsilon_{n} / 2}\right)\right\}$ (if $N_{v}=\emptyset$ we simply set $\left.f_{n}=f\right)$ for $z \in \partial D \backslash S_{v}$ and $f_{n}(z)=0$ for $z \in S_{v}$. Then $f_{n} \in C(\partial D)$ since $f_{n}$ is clearly continuous on $\partial D \backslash\left(S_{v} \cup N_{v}\right)$, zero in a neighbourhood of $N_{v}$, and $\lim _{z \rightarrow z_{0}} f(z)=0$ for $z_{0} \in S_{v}$ since $|f(z)| \leq C / v^{*}(z)$ for all $z \in \partial D \backslash\left(S_{v} \cup N_{v}\right)$ by assumption. Moreover, the previous choices yield that $\sup _{z \in \partial D}\left|f_{n}(z)-f(z)\right| v^{*}(z) \leq \sup _{z \in\left(N_{0}\right)_{e}}|\tilde{g}| \leq 1 / n$ which establishes that $f \in C^{*} v(\partial D)$.

(iii) and (iv) follow from (i).

We now study the Banach space case.

Our aim is to find conditions which imply that 
or

$$
\text { the map } f \mapsto f^{*} \text { is an isomorphism } h v_{c}(D) \rightarrow C^{*} v(\partial D) .
$$

The following characterization can be obtained by a standard argument.

Theorem 4.2. Let $v$ be a weight as in (4.1) and assume that either, for some constants $q>1$ and $C>0,\left\|1 / v_{r}\right\|_{L^{q}} \leq C$ for all $0<r<1$, or that the functions $1 / v_{r}, 0<r<1$, have a common integrable majorant. Then $(A)\left(\right.$ resp. $\left.\left(A^{\prime}\right)\right)$ holds if and only if and there is a constant $C^{\prime}>0$ such that

$$
P\left(1 / v^{*}, z\right) \leq C^{\prime} / v(z) \text { for all } z \in D .
$$

Proof. We first prove that the conditions on $v$ imply that every element of $h v(D)$ has radial boundary values and may be expressed as the Poisson extension of its radial boundary values. Assume that $f \in h v(D)$. Then $\left|f_{r}\right| \leq C / v$, for $0<r<1$ which implies that the sequence $f_{(1-1 / n)}(n \in \mathbb{N})$ is either a bounded sequence in the reflexive space $L^{q}(\partial D)$ or a uniformly integrable sequence in the space $L^{\prime}(\partial D)$. In either case we may subtract a subsequence $f_{r_{n}}$ converging weakly to $g \in L^{1}(\partial D)$. Taking the limit $n \rightarrow \infty$ in the equality $f\left(r_{n} z\right)=P\left(f_{r_{n}}, z\right)$ we see that $f(z)=P(g, z)$ for all $z$. This implies ([20, Corollary 11.12]) that $f$ has boundary values a.e. and $f^{*}=g$. Hence $f=P\left(f^{*}, \cdot\right)$.

Assume next that $(A)$ or $\left(A^{\prime}\right)$ holds. Since $g_{n}:=\min \left(n, 1 / v^{*}\right) \in C^{*} v(\partial D)(n \geq 1)$ with norm less than 1 , the assumption implies that $\left\|P\left(g_{n}, \cdot\right)\right\|_{v} \leq C^{\prime}$ for all $n$. Hence $P\left(g_{n}, z\right) v(z) \leq C^{\prime}$ for $z \in D$, and letting $n \rightarrow \infty$ the monotone convergence theorem yields $(B)$.

Conversely, assume that the condition $(B)$ holds. An easy limiting argument implies that the map $f \rightarrow f^{*}$ is an injective contraction between the corresponding spaces. The assumptions imply that $1 / v^{*} \in L^{1}(\partial D)$, hence $L^{\infty} v^{*}(\partial D) \subset L^{\prime}(\partial D)$. If $g \in C^{*} v(\partial D)$ (resp. $\left.g \in L^{\infty} v^{*}(\partial D)\right)$ has norm 1 we set $w=P(g, \cdot)$ so that $w^{*}=g$. Since the Poisson kernel is positive, we have

$$
|w(z)|=|P(g, z)| \leq P\left(1 / v^{*}, z\right) \leq C^{\prime} / v(z),
$$

hence $p_{v}(w) \leq C^{\prime}$, and the map $g \mapsto P(g, \cdot)$ is a bounded inverse of $f \rightarrow f^{*}$. The proof is completed as we note that clearly $w \in h v_{c}(D)$ for $g \in C^{*} v(\partial D)$.

Remark. There are examples which show that the sheer integrability of $1 / v^{*}$ is not enough for $(A)$ to hold even if $f^{*}$ is well defined for all $f \in h v(D)$.

Our aim is next to study the consequences of this result to weighted inductive limits.

Applying Theorem 4.2 to the step spaces gives immediately the following. 
Corollary 4.3. Let $V=\left(v_{k}\right)_{k=1}^{\infty}$ be a decreasing sequence of weights on $D$ satisfying one of the assumptions of Theorem 4.2 and the condition $(B)$. Then the spaces $\mathcal{V} h(D)$ and $\mathcal{V}^{*} L^{\infty}(\partial D)$ are isomorphic.

Theorem 4.2 and Lemma 4.1.(ii)-(iii) yield an interesting dichotomy in the behaviour of the space $\mathcal{V}_{c} h(D)$ :

Corollary 4.4. Let $V$ be as in Corollary 4.3.

(i) If $N_{v_{1}}=N_{v_{1}}$ and $S_{v_{1}}=S_{u_{2}}$ for all $k$, then the spaces $\mathcal{V}_{\mathrm{c}} h(D)$ and $\mathcal{V} C^{*}(\partial D):=$ $\operatorname{ind}_{k} C^{*}\left(v_{k}\right)(\partial D)$ are isomorphic.

(ii) If in addition $S_{v_{1}}=\emptyset$, then the spaces $\mathcal{V}_{c} h(D)$ and $\mathcal{V}_{0}^{*} C(\partial D):=\operatorname{ind}_{k} C\left(v_{k}^{*}\right)_{0}\left(\partial D \backslash N_{v_{1}}\right)$ are isomorphic.

(iii) If, in addition to (i), $N_{v_{\mathrm{t}}}=\emptyset$, then the spaces $\mathcal{V}_{c} h(D)$ and $\mathcal{V}^{*} C(\partial D):=$ ind $_{k} C v_{k}^{*}\left(\partial D \backslash S_{v_{1}}\right)$ are isomorphic.

Corollary 4.5. Let $V$ be as in Corollary 4.4.(ii). The following are equivalent:

(i) The inductive limit $\mathcal{V}_{c} h(D)$ is regular.

(ii) $\mathcal{V}_{\mathrm{c}} h(D)$ is complete.

(iii) $V^{*}:=\left(v_{k}^{*}\right)_{k=1}^{\infty}$, restricted to $\partial D \backslash N_{v_{1}}$, is regularly decreasing in the sense of [11, Definition 2.1].

Corollary 4.5 follows from Corollary 4.4.(ii) and Theorem 2.6 of [11].

Some examples are presented in 4.9 .

To treat the associated weighted spaces we need to prove a technical lemma.

Lemma 4.6. Let $V=\left(v_{k}\right)_{k \in \mathbb{N}}$ be a decreasing sequence of weights on $D$ such that $N_{0}:=N_{v_{1}}=N_{v_{k}}$ and $S_{0}:=S_{v_{1}}=S_{v_{k}}$ for all $k$ and such that

(i) every $v_{k}$ satisfies the condition $(B)$ with the same constant $C^{\prime}$,

(ii) $\exists q>1$ s.t. $\forall k \in \mathbb{N} \exists c_{k}^{\prime}>0$ s.t. $\forall 0<r<1$ :

$$
\left\|1 /\left(v_{k}\right)_{r}\right\|_{L^{\cdot}}<c_{k}^{\prime} \text {. }
$$

Then, for every $\bar{v} \in \bar{V}$ there exists $\bar{w} \in \bar{V}, \bar{w} \geq \bar{v}$, which has a continuous extension $\bar{D} \rightarrow[0, \infty]$, and satisfies, for a constant $C>0,\left\|1 / \bar{w}_{r}\right\|_{L^{a}}<C$ for all $0<r<1$, and satisfies the condition $(B)$.

Proof. For all $k \in \mathbb{N}$, let the numbers $C_{k}>0$ be such that

$1^{\circ} \bar{v} \leq C_{k} v_{k}$

$2^{\circ} \sup _{|z| \leq 1-1 / k}\left|1 / v_{k}(z)\right|<C_{k}$,

$3^{\circ} \quad C_{k} \geq \sup \left\{\left(v_{k}(z)\right)^{-1} \mid \operatorname{dist}\left(z, N_{v_{k}}\right) \geq 1 / k\right\}=: c_{k}^{\prime \prime}$,

$4^{\circ} \quad C_{k} \geq c_{k}^{\prime}$ for all $k$.

Set

$$
\bar{w}(z):=1 / \sum_{k} 2^{-k} C_{k}^{-1} v_{k}(z)^{-1}
$$


Now $2^{\circ}$ implies that the sum in (4.4) converges for every $z \in D$. By definition, $\bar{w} \in \bar{V}$. We also have, by $1^{\circ}$,

$$
\bar{w} \geq\left(\sup _{k} C_{k}^{-1} v_{k}(z)^{-1}\right)^{-1}=\inf _{k} C_{k} v_{k}(z) \geq \bar{v}
$$

The estimate for the $L^{q}$-norm follows from $4^{\circ}$ and the condition (B) from (i) as we show that $\bar{w}$ admits a continuous extension (again defined through (4.4)) to the boundary with values in $[0, \infty]$. In order to prove this it is enough to prove the corresponding statement for $1 / \bar{w}$. Let $z_{0} \in \partial D$. If $z_{0} \in \partial D \backslash N_{0}$ the series $\sum_{k} 2^{-k} C_{k}^{-1} v_{n}(z)^{-1}$ converges uniformly in a neighbourhood of $z_{0}$ by $3^{\circ}$, which implies the continuity at $z_{0}$. If $z_{0} \in N_{0}$, it follows that $\lim _{z \rightarrow z_{0}} v_{k}(z)=\infty$. This clearly implies that $\lim _{z \rightarrow z_{0}} 1 / \bar{w}(z)=\infty$ and again the continuity at $z_{0}$ follows.

Theorem 4.7. Let the weight sequence $V=\left(v_{k}\right)$ be as in Lemma 4.6.

(i) The spaces $h \bar{V}(D)$ and $L^{\infty} \overline{V^{*}}(\partial D)$ are isomorphic to each other.

(ii) If $V$ satisfies the conditions in Corollary 4.4.(ii), then also the spaces $h_{c} \bar{V}(D)$ and $C \overline{V^{*}}{ }_{0}\left(\partial D \backslash N_{0}\right)$ are isomorphic.

(iii) If $V$ satisfies the conditions in Corollary 4.4.(iii), then also the spaces $h_{c} \bar{V}(D)$ and $C \overline{V^{*}}\left(\partial D \backslash S_{0}\right)$ are isomorphic.

Proof. It is enough to verify that the map $f \rightarrow f^{*}$ defines the desired isomorphisms (cf. $(A)$ and $\left(A^{\prime}\right)$ ). First, by Lemma $4.6, \bar{V}$ contains weights satisfying the conditions of Theorem 4.1, including the condition $(B)$. As in the proof of Theorem 4.2 we see that every $f \in h \bar{V}(D)$ has radical boundary values a.e..

Using the proof of Theorem 4.2 ("condition $(B) \Rightarrow(A)$ and $\left(A^{\prime}\right)$ ") one can now prove (i) and the isomorphism of $h_{c} \tilde{V}(D)$ and the space $C^{*} \tilde{V}(\partial D)$; the latter space is defined as the restriction to $\partial D$ of the closed subspace of $L^{\infty} \bar{V}(D)$ which is spanned by continuous functions having continuous extensions to $\partial D$. (Compare to Lemma 4.1 (i) - here one has to use Lemma 4.6 and the following fact: given an arbitrary $\bar{w} \in \overline{V^{*}}$ there exists $\bar{v} \in \bar{V}$ such that $\bar{v}^{*} \geq c \bar{w}$ for a constant $c>0$.) Finally, as in Lemma 4.1 we have the following characterization: $f \in C^{*} \bar{V}(\partial D)$ if and only if, (modulo sets of measure zero) for all $\bar{v} \in \bar{V}$, $\left.(\bar{v} f)\right|_{\partial D \backslash\left(S, u N_{,}\right)}$is continuous and bounded and $\lim _{z \rightarrow z_{*}}(\bar{v} f)(z)=0$ for all $z_{0} \in N_{0}$. From this $C^{*} \bar{V}(\partial D)$ is easily seen to be isomorphic to $C \bar{V}_{0}^{*}\left(\partial D \backslash N_{0}\right)$ in case (ii) or to $C^{*} \bar{V}\left(D \backslash S_{0}\right)$ in case (iii).

Unfortunately it seems that a characterization of the bornologicity of $L^{\infty} \bar{V}$-spaces is not yet generally available in the literature. Philosophically it is probable that this should be possible to obtain using the same methods as in the case of spaces of continuous functions. In the case of spaces with continuous boundary values we get the following result, by combining Theorem 4.7 with Corollary 4.3 and using the known results on weighted inductive limits of spaces of continuous mappings, see [11, Theorem 2.6], and [10], [2], [7]. 


\section{WEIGHTED SPACES OF HARMONIC AND HOLOMORPHIC FUNCTIONS 59}

Corollary 4.8. Let $V$ be as in Lemma 4.6.

(i) If $V$ satisfies the conditions in Corollary 4.4.(ii), then $\mathcal{V}_{c} h(D)$ is isomorphic to a dense subspace of $h \bar{V}_{c}(D)$. These spaces are isomorphic if and only if $V^{*}$ is in addition regularly decreasing.

(ii) If $V$ satisfies the conditions in Corollary 4.4.(iii), then $h \bar{V}_{c}(D)$ is bornological if and only if $V^{*}$ satisfies the condition $(D)$. If this is the case, then $h \bar{V}_{c}(D)$ is isomorphic to $\mathcal{V}_{c} h(D)$, and moreover, $\mathcal{V}_{c} h(D) \subset \mathcal{V} h(D) \subset \mathcal{V} C(D)$ holds as topological subspaces.

We devote the rest of this section to analyze the isomorphisms $(A)$ and $\left(A^{\prime}\right)$ and the condition $(B)$ more closely. The following proposition gives necessary and sufficient conditions for the isomorphisms in the case that the weight function has only one zero or singularity that is approached monotonically.

Proposition 4.9. Assume that $v(z)=1 / g(|1-z|)$, where $g:[0,2] \rightarrow[0, \infty]$ is monotonic, continuous and satisfies $0<g(t)<\infty$ for $t \in] 0,2]$.

(i) If $g(0)=0$, then $(A)\left(\right.$ resp. $\left.\left(A^{\prime}\right)\right)$ holds if and only if there is a constant $C>0$ such that

$$
\begin{gathered}
g(2 t) \leq C g(t) \quad \text { for } 0 \leq t \leq 1 \text { and } \\
\left.\left.\int_{t}^{2} x^{-2} g(x) d x \leq C t^{-1} g(t) \quad \text { for all } t \in\right] 0,2\right] .
\end{gathered}
$$

(ii) In the case $g(0)=\infty,(A)$ (resp. $\left(A^{\prime}\right)$ ) holds if and only if $g$ is integrable and there is a constant $C>0$ such that

(C') $g(t) \leq C g(2 t)$ for $0 \leq t \leq 1$ and $\int_{0}^{t} g(x) d x \leq C t g(t)$ for all $\left.\left.t \in\right] 0,2\right]$.

Proof. (i) In this case $g(t)$ decreases monotonically to 0 as $t \rightarrow 0^{+}$. Now $1 / v$ is bounded from above and hence by Theorem 4.2 it is enough to check the condition $(B)$. Suppose first that $(C)$ holds. We must show that

$$
P\left(g\left(\left|1-e^{i 0}\right|, z\right) \leq C g(|-z|)\right) .
$$

By continuity it is enough to consider $z$ with $r:=|1-z| \leq 1 / 10$. Write

$$
P\left(g\left(\left|1-e^{i \theta}\right|\right), z\right)=P\left(g\left(\left|1-e^{i \theta}\right|\right) \chi_{[-2 r, 2 r]}(\theta), z\right)+P\left(g\left(\left|1-e^{i \theta}\right|\right)\left(1-\chi_{[-2 r, 2 r]}(\theta)\right), z\right)=: u_{1}+u_{2},
$$

where $\chi_{[a, b]}$ is the characteristic function of $[a, b]$. In this decomposition $u_{1}$ is a bounded harmonic function with $\left\|u_{1}^{*}\right\|_{\infty} \leq g\left(\left|1-e^{2 i r}\right|\right) \leq g(2 r)$, where the elementary geometric estimate $\left|1-e^{2 i r}\right| \leq 2 r$ was applied. Thus $\left|u_{1}(z)\right| \leq C g(r)=C g(|1-z|)$.

In order to estimate $u_{2}$ we note that $\left|z-e^{i 0}\right| \geq\left|1-e^{i 0}\right| / 3=2 \sin (|0| / 2) / 3$ for $2 r \leq|\theta| \leq \pi$. This observation and the substitution $2 \sin |\theta / 2|=s$ for $2 r \leq|\theta| \leq \pi$ in the Poisson formula $u_{2}(z)=\left(1-|z|^{2}\right)(2 \pi)^{-1} \int_{2 r \leq|0| \leq \pi} g\left(\left|1-e^{i \theta}\right|\right)\left|z-e^{i \theta}\right|^{-2} d \theta$ leads to

$$
u_{2}(z) \leq \frac{18\left(1-|z|^{2}\right)}{2 \pi} \int_{2 \sin r}^{2} \frac{g(s) d s}{s^{2} \sqrt{1-s^{2} / 4}} .
$$


We note that $1-|z|^{2}=(1-|z|)(1+|z|) \leq 2 r$ and $2 \sin r \geq r$. By writing $C_{1}=$ $\int_{1}^{2} g(s) s^{-2}\left(1-s^{2} / 4\right)^{-1 / 2} d s /\left(\int_{1}^{2} g(s) s^{-2} d s\right)$ and using $(C)$ we obtain the desired estimate

$$
u_{2}(z) \leq\left(2 / \sqrt{3}+C_{1}\right) \cdot 18 \cdot \frac{2 r}{2 \pi} \int_{r}^{2} \frac{g(s) d s}{s^{2}} \leq C_{2} g(r)=C_{2} g(|1-z|),
$$

which was to be shown.

In order to prove the converse assume that (4.5) holds. By choosing in (4.5) $z=1-r$ with $0<r \leq 1 / 10$ we have $1-|z|^{2}=r(2-r) \geq r$ and

$$
C g(r) \geq P\left(g\left(\left|1-e^{i 0}\right|\right), 1-r\right) \geq \frac{1}{2 \pi} \int_{3 r}^{4 r} \frac{r g\left(\left|1-e^{i 0}\right|\right) d \theta}{\left|(1-r)-e^{i 0}\right|^{2}}
$$

Elementary geometry yields $\left|(1-r)-e^{i 0}\right| \leq 4 r$ and $\left|1-e^{i 0}\right| \geq 2 r$ for $\theta \in[3 r, 4 r]$. By substituting these estimates in (4.7) we get

$$
C g(r) \geq \frac{1}{2 \pi} \frac{r}{16 r^{2}} g(2 r) r=C^{\prime} g(2 r)
$$

for $r \in] 0,1 / 10]$. By continuity $g(2 r) \leq C^{\prime \prime} g(r)$ for all $r \in[0,1]$.

Next we apply the geometric estimate $\left|1-r-e^{i 0}\right| \leq 2\left|1-e^{i 0}\right|$ for $|\theta| \in[2 r, \pi]$ (remember that $r \in] 0,1 / 10]$ ) and obtain (compare with the proof of (4.6))

$$
P\left(g\left(\left|1-e^{i 0}\right|\right), 1-r\right) \geq \frac{1}{4} \cdot \frac{r}{2 \pi} \int_{2 \sin r}^{2} \frac{g(s) d s}{s^{2} \sqrt{1-s^{2} / 4}} \geq \frac{r}{8 \pi} \int_{2 r}^{2} \frac{g(s) d s}{s^{2}} .
$$

Combining this with the assumption and the monotonicity of $g$ leads to

$$
g(r) \geq g(r / 2) \geq \frac{1}{C} P\left(g\left(\left|1-e^{i 0}\right|\right), 1-r / 2\right) \geq c^{\prime} r \int_{r}^{2} \frac{g(s) d s}{s^{2}} .
$$

Thus the other half of $(C)$ holds as well.

(ii) In this case $g(t)$ increases monotonically to $\infty$ as $t \rightarrow 0^{+}$. Assume first that $g$ is integrable and $\left(C^{\prime}\right)$ holds. An easy geometric exercise shows that

$$
\sup _{0 \leq r<1} 1 / v\left(r e^{i \theta}\right)=1 / v\left(\cos (\theta) e^{i \theta}\right)=g(|\sin \theta|) \leq g(|\theta| / 2)
$$

for $\theta \in[-\pi / 2, \pi / 2] \backslash\{0\}$. Thus the functions $1 / v_{r}(0 \leq r<1)$ have a common integrable majorant and Theorem 4.2 shows that it is enough to verify that $(B)$ holds. To that end we assume again that $z \in D$ with $r=|z-1| \leq 1 / 10$ and write

$$
P\left(g\left(\mid 1-e^{i 0 \mid}\right), z\right)=P\left(g\left(\left|1-e^{i 0}\right|\right)\left(1-\chi_{[-r / 2, r / 2]}(0)\right), z\right)+P\left(g\left(\left|1-e^{i 0}\right|\right) \chi_{[-r / 2, r / 2]}(\theta), z\right)=: u_{1}+u_{2}
$$


As before we deduce that $\left|u_{1}(z)\right| \leq g\left(\left|1-e^{i r / 2}\right|\right) \leq g(r / 4) \leq C^{2} g(r)$, where we applied the first condition of $\left(C^{\prime}\right)$. Moreover, $\left(1-|z|^{2}\right) \leq 2 r,\left|1-e^{i \theta}\right| \geq 0 / 2$ and $\left|z-e^{i \theta}\right| \geq r / 4$ for $\theta \in[-r / 2, r / 2]$ so that

$$
u_{2}(z) \leq \frac{1}{2 \pi} \int_{-r / 2}^{r / 2} \frac{r g(\theta / 2) d \theta}{r^{2} / 16}=\frac{64}{\pi r} \int_{0}^{r / 4} g(\theta) d \theta \leq \frac{16}{\pi} C g(r / 4) \leq \frac{16}{\pi} C^{3} g(r),
$$

where we used both conditions of $\left(C^{\prime}\right)$.

In order to obtain the converse statement assume that the isomorphism $(A)$ (resp. $\left.\left(A^{\prime}\right)\right)$ holds. Note first that $1 / v \geq g(2)$ and hence $h v_{c}(D)$ contains all bounded harmonic functions. In particular, $u_{n} \in h v_{c}(D)$, where $u_{n}=P\left(\min \left(n, 1 / v^{*}\right), \cdot\right)$ for integers $n \geq 1$. Clearly $\left\|u_{n}^{*}\right\|_{v^{*}} \leq 1$ and hence $u_{n}(0) \leq C$ for all $n \geq 1$. Letting $n \rightarrow \infty$ in the representation $u_{n}(0)=(2 \pi)^{-1} \int_{\partial D} \min \left(n, 1 / v^{*}\right)$ we see that $1 / v^{*}$ and hence $g$ is integrable.

Thus the functions $1 / v_{r}(0<r<1)$ have a common integrable majorant and Theorem 4.2 implies that $(B)$ holds. The rest of the proof is analogous to what we did before: The inequality $g(r / 2) \leq C g(r)$ is deduced by comparing $g(r)=1 / v(1-r)$ and $P\left(g\left(\left|1-e^{i 0}\right|\right) \chi_{[0, r / 4]}, 1-r\right)$, and the estimate $g(r) \geq r^{-1} \int_{0}^{r} g(t) d t$ is obtained by comparing $g(r)=1 / v(1-r)$ and $P\left(g\left(\left|1-e^{i 0}\right|\right) \chi_{[0,2 r]}, 1-r\right)$.

Now we can close this section with some

4.10. Examples. (i) For $\alpha \in \mathbb{R}$ and weights $v=|1-z|^{\alpha}$ or $v=\log (3 /|1-z|)|1-z|^{x}$ the isomorphisms $(A)$ and $\left(A^{\prime}\right)$ hold if and only if $\left.\alpha \in\right]-1,1[$, as is easily verified by applying Proposition 2.

(ii) The system $V:=\left(v_{k}\right)_{k=1}^{\infty}$, where $v_{k}(z):=|1-z|^{\alpha k /(1+k)}$ and $0<\alpha<1$, satisfies the assumptions of Lemma 4.6 and Corollary 4.4.(ii). Moreover, $V^{*}$ is regularly decreasing. Hence, Corollaries 4.3, 4.4.(i)-(ii), 4.4 and 4.8.(i)-(ii) and Theorem 4.7.(i)-(ii) apply.

(iii) Similarly, the system $\left(v_{k}\right)_{k=1}^{\infty}(z):=\left(|1-z|^{-1 /(k+1)}\right)_{k=1}^{\infty}$ satisfies the assumptions in Lemma 4.6 and Corollary 4.4.(iii), and $\left(v_{k}^{*}\right)$ satisfies the condition $D$. This is an example where Corollaries 4.3, 4.4.(i), (iii), 4.5 and 4.8.(i), (ii) and Theorem 4.7.(i), (iii) work.

\section{REFERENCES}

1. L. Ahlfors, Complex analysis, 3rd ed. (McGraw-Hill, New York, 1979).

2. F. BASTIn, On bornological spaces $C \bar{V}(X)$, Archiv. Math. 53 (1989), 393-398.

3. C. A. Berenstein and M. A. Dostal, Analytically Uniform Spaces and their Applications to Convolution Equations (Springer Lecture Notes in Math. 256, 1972).

4. K. D. Bierstedt, Weighted inductive limits of spaces of holomorphic functions, in Proceedings of the 23rd Annual Iranian Congress of Mathematics, April 1992, to appear.

5. K. D. BierstedT and J. Bonet, Stefan Heinrich's density condition for Fréchet spaces and the characterization of the distinguished Köthe echelon spaces. Math. Nachr. 135 (1988), 149-180.

6. K. D. Bierstedt and J. Bonet, Dual density conditions in (DF)-spaces, I. Resultate Math. 14 (1988), 242-274. 
7. K. D. Bierstedt and J. Bonet, Dual density conditions in (DF)-spaces, II. Bull. Soc. Roy. Sci. Liége 57 (1988), 567-589.

8. K. D. Bierstedt and J. Bonet, Some recent results on $\mathcal{V} C(X)$, in Advances in the theory of Fréchet spaces (Kluwer, 1989), 181-194.

9. K. D. BIERSTEDT and R. MeiSE, Weighted inductive limits and their projective descriptions, Doga Mat. 10,1 (1986), 54-82. (Special issue: Proceedings of the Silivri Conference 1985).

10. K. D. Bierstedt and R. Meise, Distinguished echelon spaces and the projective description of weighted inductive limits of type $\mathcal{V}_{d} C(X)$, in Aspects of Mathematics and its Applications (Elsevier, 1986), 169-226.

11. K. D. Bierstedt, R. Meise and W. H. Summers, A projective description of weighted inductive limits, Trans. Amer. Math. Soc. 272 (1982), 107-160.

12. K. D. Bierstedt, R. Meise and W. H. Summers, Köthe sets and Köthe sequence spaces, Functional Analysis, Holomorphy and Approximation Theory (North-Holland Math. Studies 71, 1982), 27-91.

13. J. Bonet and J. TASKInEN, The subspace problem for weighted inductive limits of spaces of holomorphic functions, in Reports of the Department of Mathematics, University of Helsinki, 51 (1994).

14. L. EhrenPreIS, Fourier Analysis in Several Complex Variables (Interscience Tracts in Math. 17, Wiley, 1970).

15. J. HoR VÁth, Topological Vector Spaces and Distributions (Addison-Wesley, 1966).

16. Koosis, Introduction to $H^{p}$ spaces (Cambridge University Press, 1980).

17. G. KöтнE, Topological vector spaces, Vol. 1, Second printing (Springer Verlag, 1983).

18. W. LUSKY, On weighted spaces of harmonic and holomorphic functions, J. London Math. Soc., to appear.

19. P. Pérez Carreras and J. Bonet, Barrelled Locally Convex Spaces (North-Holland Math. Studies 131, 1987).

20. W. Rudin, Real and complex analysis, second edition (McGraw-Hill, New York, 1974).

21. A. Shields and D. Williams, Bounded projections, duality and multipliers in spaces of harmonic functions, J. Reine Angew. Math. 299/300 (1978), 259-279.

22. B. A. TAYLOR, A seminorm topology for some (DF)-spaces of entire functions, Duke Math. J. 38 (1971), 379-385.

23. D. VoGt, Distinguished Köthe spaces, Math. Z. 202 (1989), 143-146.

Department of Mathematics

P.O. BOX 4 (Hallituskatu 15)

FIN-00014 UNIVERSITY OF HELSINKI

FINLAND 